\title{
NUMERICAL SIMULATION OF HEAT AND MASS TRANSFER IN A LIQUID FILM MOVING OVER A HEATED HORIZONTAL SURFACE UNDER THE ACTION OF A GAS FLOW
}

\author{
Maria V. Bartashevich ${ }^{1,{ }^{*}}$, Maxim G. Vlasenko ${ }^{1}$, Andrey A. Pil'nik ${ }^{1}$, and Andrey A. \\ Chernov $^{1}$ \\ ${ }^{1}$ Kutateladze Institute of Thermophysics, 630000 Novosibirsk, Russia
}

\begin{abstract}
Heat and mass transfer during desorption on a horizontal film of lithium bromide water solution flowing on a heated wall under the action of shear stress is numerically investigated in this paper. The shear stress on the film surface is set by the motion of surrounding saturated water vapor. It is shown that at low values of heat flux the film temperature and vapor concentration in the solution downstream decreases due to desorption. However, with an increase in heat flux, general film heating and desorption slowing down are observed.
\end{abstract}

\section{Problem statement}

In the paper, combined heat and mass transfer in the process of desorption on a liquid film moving on a heated horizontal substrate under the action of shear stress is numerically investigated. The liquid film is a water solution of lithium bromide, the shear stress on the film surface is set by the motion of surrounding saturated water vapor. Desorption takes place in various energy devices, for example, in the absorption heat pump, consisting of generator (desorber), condenser, evaporator, and absorber [1,2]. It is assumed that the shear stress applied to the interface will allow intensification of the process of heat and mass transfer in comparison with the fixed-vapor regime. The investigation of thin liquid film flowing down a vertical wall in the roll-wave regime in presence of heat and mass transfer through the free surface was presented in $[3,4]$. The absorption and desorption processes under the conditions, corresponding to the operating modes of thermal transformers are described in [5]. The analytical solution to the problem of conjugate heat and mass transfer in a laminar falling liquid film with a linear velocity profile is presented in [6]. The results of the numerical simulation of absorption and their comparison with the experimental data are presented in [7].

We consider the two-dimensional stationary flow of a laminar liquid film of thickness $h$ over a horizontal plate. The surface of liquid is in contact with moving vapor. The shear

\footnotetext{
Corresponding author: bartashevichmv@gmail.com
} 
stress on the film surface is $\tau$. Desorption is considered in the framework of ordinary assumptions [1]. The thermal-physical properties of solution are assumed constant.

Let us introduce the Cartesian coordinate system with $O x$ axis in the direction of the flow and $O y$ axis directed along the normal to the plate. The velocity profile in the film takes form

$$
u=y \tau / \mu .
$$

The process of heat and mass transfer at film desorption is described by the equations of heat conductivity and diffusion:

$$
\begin{aligned}
& u \partial T / \partial x=a \partial^{2} T / \partial y^{2} \\
& u \partial C / \partial x=D \partial^{2} C / \partial y^{2}
\end{aligned}
$$

$a$ is thermal diffusivity, $\mathrm{m}^{2} / \mathrm{s} ; D$ is diffusion coefficient, $\mathrm{m}^{2} / \mathrm{s}$. The boundary conditions at the inlet at $x=0$ are:

$$
T=T_{i}, C=C_{i} .
$$

Similarly to [1], the equilibrium of solution-vapor system occurs on the interface at $y=h$ :

$$
C_{d}=f\left(T_{d}\right),-\lambda \partial T / \partial y=-\chi \partial C / \partial y
$$

where $\chi=L \rho D /\left(1-C_{i}\right), L$ is latent heat of phase transformation, $\mathrm{J} / \mathrm{kg} ; \rho$ is solution density, $\mathrm{kg} / \mathrm{m}^{3} ; \lambda$ is heat conductivity, $\mathrm{W} /(\mathrm{mK})$. This equilibrium condition joins equilibrium temperature $T_{d}$ with concentration $C_{d}$. We set on the wall at $y=0$ :

$$
-\lambda \partial T /\left.\partial y\right|_{y=0}=q_{w}, \partial C /\left.\partial y\right|_{y=0}=0,
$$

where $q_{w}$ is heat flux on the wall. Let us turn to dimensionless variables $\xi=x / l$, $l=\tau h^{3} /(\mu a), \quad \eta=y / h, \quad \theta=\left(T-T_{i}\right) /\left(T_{s}-T_{i}\right) \quad$ and $T_{s}<T_{i}, \quad \gamma=\left(C-C_{i}\right) /\left(C_{s}-C_{i}\right)$ and $C_{s}<C_{i}$ under the conditions of desorption. Here, $C_{s}=f\left(T_{i}\right)$ is equilibrium concentration, corresponding to initial temperature of solution $T_{i} ; T_{s}$ is equilibrium temperature corresponding to initial concentration $C_{i}\left(C_{i}=f\left(T_{s}\right)\right)$, similarly to [1]. The system of equations (1) - (2), reduced to the dimensionless form, is rewritten in the following way:

$$
\begin{aligned}
& \eta \partial \theta / \partial \xi=\partial^{2} \theta / \partial \eta^{2}, \\
& \eta \partial \gamma / \partial \xi=\operatorname{Le} \partial^{2} \gamma / \partial \eta^{2},
\end{aligned}
$$

here $\mathrm{Le}=D / a$. The boundary conditions at the inlet for $\xi=0$ :

$$
\theta=0, \gamma=0 \text {. }
$$

Similarly to [1], on the interface at $\eta=1$ and linear character of function $f(T)=k_{1}-k_{2} T$, we obtain:, similarly to [1]: 


$$
\begin{gathered}
\theta=\theta_{d}, \gamma=\gamma_{d}, \\
\theta_{d}+\gamma_{d}=1,\left(\operatorname{KuLe}\left(C_{s}-C_{i}\right) /\left(1-C_{i}\right)\right) \partial \gamma / \partial \eta=\partial \theta / \partial \eta .
\end{gathered}
$$

Here $\mathrm{Ku}=L /\left(\mathrm{C}_{p}\left(\mathrm{~T}_{s}-\mathrm{T}_{i}\right)\right)$ is Kutateladze number; $\mathrm{C}_{p}$ is specific heat capacity of solution, $\mathrm{J} /(\mathrm{kg} / \mathrm{K})$. On the wall at $\eta=0$, we obtain:

$$
\partial \theta /\left.\partial \eta\right|_{\eta=0}=-\bar{q}_{w}, \partial \gamma /\left.\partial \eta\right|_{\eta=0}=0
$$

where $\bar{q}_{w}$ is dimensionless heat flux.

\section{Numerical calculations}

Numerical calculations were carried out by the finite difference method. The results of calculations of desorption on a film moving under the action of a gas flow over a horizontal wall are given in Fig.1 - Fig.4. The calculations were performed at $\left(C_{s}-C_{i}\right) /\left(1-C_{i}\right)=$ $-0.4, \mathrm{Ku}=-25, \mathrm{Le}=0.01$ and $\bar{q}_{w}$ from 0.01 to 0.3 .

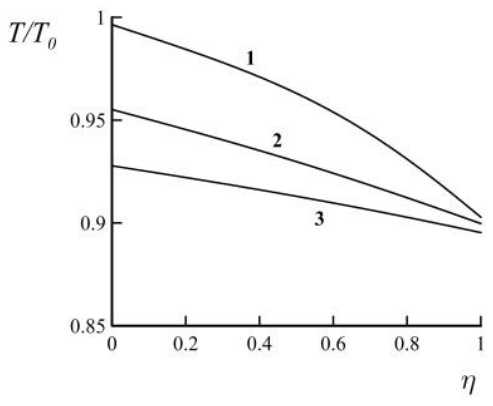

Fig. 1. Profiles of dimensionless temperature in different cross-sections of the film, $\bar{q}_{w}=0.05 .1-\xi$ $=0.1 ; 2-\xi=0.5 ; 3-\xi=1.0$.

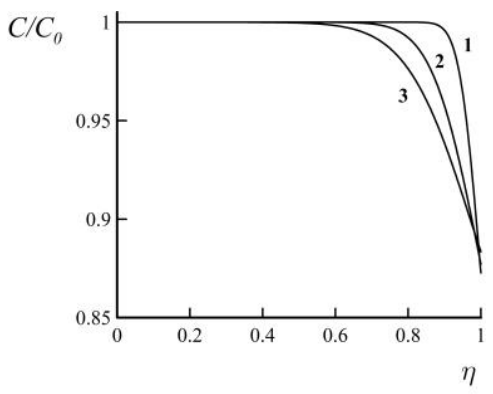

Fig. 2. Profiles of dimensionless concentration in different cross-sections of the film, $\bar{q}_{w}=0.05 .1-$ $\xi=0.1 ; 2-\xi=0.5 ; 3-\xi=1.0$.

Figure 1 shows the calculated distributions of the dimensionless temperature at different distances from the inlet, and in Fig. 2 there are corresponding concentration distributions. Figure 3 shows the calculated distributions of the dimensionless temperature along the film surface for different heat fluxes on the wall. Corresponding concentration distributions vs. longitudinal coordinate are shown in Fig. 4. 


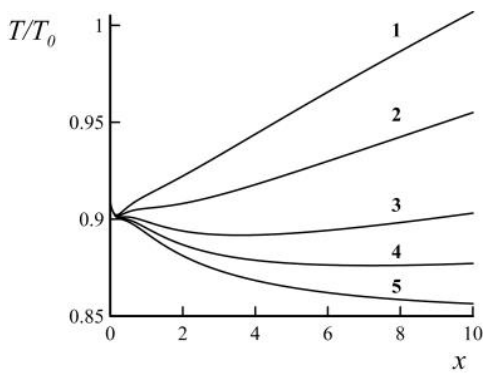

Fig. 3. Profiles of dimensionless temperature of the film surface at different heat fluxes: $1-\bar{q}_{w}=0.3 ; 2-\bar{q}_{w}=0.2 ; 3-\bar{q}_{w}=0.1 ; 4-\bar{q}_{w}=0.05 ; 5-\bar{q}_{w}=0.01$.

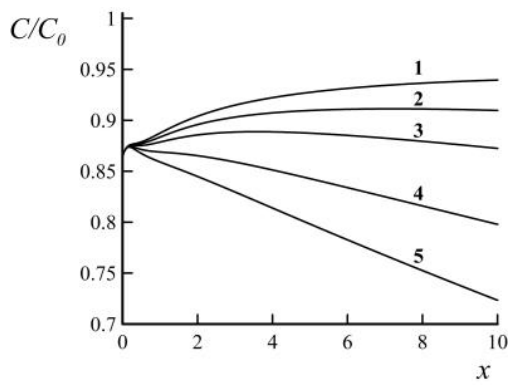

Fig. 4. Profiles of dimensionless concentration on the film surface at different heat fluxes: $1-\bar{q}_{w}=0.3 ; 2-\bar{q}_{w}=0.2 ; 3-\bar{q}_{w}=0.1 ; 4-\bar{q}_{w}=0.05 ; 5-\bar{q}_{w}=0.01$.

\section{Conclusions}

The problem of combined heat and mass transfer at film desorption on a liquid film moving over the heated horizontal substrate under the action of shear stress for the given constant heat flux is numerically investigated. It is shown that at low values of $\bar{q}_{w}$ the film temperature and vapor concentration in the solution downstream decreases due to desorption. However, with an increase in $\bar{q}_{w}$, general film heating and desorption slowing down are observed.

This work was carried out at the Kutateladze Institute of Thermophysics SB RAS and financially supported by the Russian Science Foundation (project number 15-19-10025).

\section{References}

1. V.E.Nakoryakov, N.I.Grigorieva, Non-Isothermal Absorption in Thermal Transformers (Nauka, Novosibirsk, 2010)

2. V.E. Nakoryakov, N.I. Grigoryeva, M.V. Bartashevich, Int. J. Heat and Mass Transfer, 54, 21 (2011)

3. V.E. Nakoryakov, V.V. Ostapenko, M.V.Bartashevich, Int. J. Heat and Mass Transfer, 55, 23 (2012)

4. V.E. Nakoryakov, V.V. Ostapenko, M.V.Bartashevich, Int. J. Heat and Mass Transfer, 89 (2015)

5. M. Mittermaier, F. Ziegler, Int. J. Refrig, 59, (1) (2015)

6. M. Mortazavi, S. Moghaddam, Int. J. Refrig, 66 (2016)

7. E. Garcia-Rivera, J. Castro, J. Farnos, A. Oliva, Int. J. Thermal Sciences, 109 (2016) 